\title{
PATOLOGÍA INFLAMATORIA EN UROLOGÍA. SISTEMATIZACIÓN
}

\author{
J.A. CARBALLIDO*, M. ÁLVAREZ-MON**, C. OLIVIER*** \\ *Hospital Universitario Puerta de Hierro. Departamento de Cirugía. Universidad Autónoma de Madrid. \\ **Hospital Universitario Príncipe de Asturias. ${ }^{* * *}$ Departamento de Medicina. Universidad de Alcalá. Madrid.
}

Actas Urol Esp. 27 (3): 173-179, 2003

\section{RESUMEN}

"PATOLOGÍA INFLAMATORIA EN UROLOGÍA. SISTEMATIZACIÓN"

Uno de los campos de expansión de la Medicina en los últimos años, es el que se refiere al reconocimiento, identificación y tratamiento de los múltiples acontecimientos que ocurren en relación con la respuesta inflamatoria. En este sentido, los avances alcanzados en los últimos años en el estudio de los mecanismos moleculares y celulares de la reacción inflamatoria tisular en diversas localizaciones como son la membrana sinovial, la mucosa intestinal, el endotelio vascular, etc... se consideran muy relevantes.

Los autores sensibilizados por estos acontecimientos efectúan una sistematización de los modelos clínicos de respuesta inflamatoria de los órganos genitourinarios en respuesta a la acción de noxas variadas. La elevada frecuencia y morbimortalidad de la patología inflamatoria, así como el difícil ejercicio de integrar a la Urología conocimientos que se desarrollan con gran rapidez en otras áreas de las Ciencias Biomédicas justifican esta revisión.

PALABRAS CLAVE: Inflamación. Patología inflamatoria. Inflamación en órganos génitourinarios.

\section{ABSTRACT \\ "INFLAMMATORY DISEASE IN UROLOGY. STANDARDIZATION"}

The recognition, identification and management of the many events taking place in relation to an inflammatory response has become a rapidly growing field in medicine over the last few years. In this sense, the progress made in later years in the study of the molecular and cellular mechanisms of tissue inflammatory reactions in sites such as synovial membranes, gut mucosa, vascular endothelium etc, is considered highly relevant.

A number of authors conscious of such events work on the standardization of clinical models of the inflammatory responses of genitourinary organs resulting from the action of a variety of harmful events. This review is warranted by the prevalence and morbidity and mortality of inflammatory conditions, as well as the complex work of incorporating to Urology the findings from other Biomedical Sciences.

KEY WORDS: Inflammation. Inflammatory diseases. Genitourinary organs inflammation. 


\section{INFILTRACIÓN INFLAMATORIA TISULAR}

La respuesta inflamatoria de los distintos organismos contribuye a la conservación de la homeostasis y de su equilibrio metabólico. En este sentido, se trata de una respuesta de carácter adaptativo frente a la agresión de diversas noxas o agresiones cuya naturaleza y origen es muy diversa. El objetivo final es la integridad orgánica y entre sus elementos fisiológicos se reconocen, por ejemplo, estructuras físicas como la queratina, los protones, el moco o, en el aparato urinario, los glucosaminoglicanos del revestimiento urotelial. En contraposición, la agresión puede tener su origen en el exterior y estar representada por agentes físicos, químicos o biológicos, o por el contrario, en el propio interior de la economía con énfasis especial en los productos del catabolismo y en las transformaciones neoplásicas.

La naturaleza de la respuesta inflamatoria es compleja y sus bases celulares y moleculares condicionan que su comprensión y traducción clínica posean hechos comunes y diferenciales en base fundamentalmente a las características del agente etiológico, al patrón de predominio celular de la respuesta inducida, de la secreción de patrones diferenciales de citocinas o quimioquinas y, finalmente, de la acción directa del agente etiológico.

El sistema de defensa constituido por las células inmunológicas e inflamatorias poseen una relevancia especial como mecanismo de defensa frente a la agresión, y en el desarrollo de la respuesta de naturaleza inflamatoria la participación de estos componentes celulares y moleculares es, en muchos casos, compartido y con implicaciones mutuas a través de la secreción de diversos factores solubles que contribuyen a su regulación funcional. Por este motivo, la distinción entre sistema inmune y respuesta inflamatoria no tiene fundamento.

Un acontecimiento primordial y precoz del fenómeno inflamatorio es la modificación de la fisiología celular, la cual es variable de acuerdo al tipo de agresión, a su intensidad y a su mayor o menor mantenimiento temporal (Tabla I y Tabla II). La naturaleza de la modificación observada en la funcionalidad celular desencadena una reacción de stress o incluso muerte celular. Estos fenómenos traducen, fundamentalmente, modifica-
TABLA I

INFLAMACIÓN EN ÓRGANOS GENITOURINARIOS

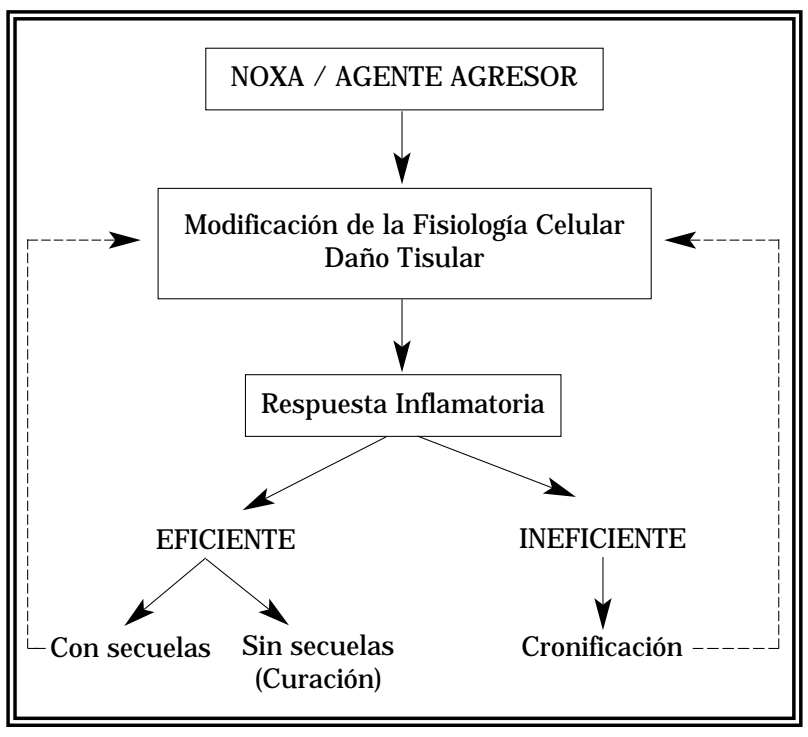

TABLA II

INFLAMACIÓN EN ÓRGANOS GENITOURINARIOS

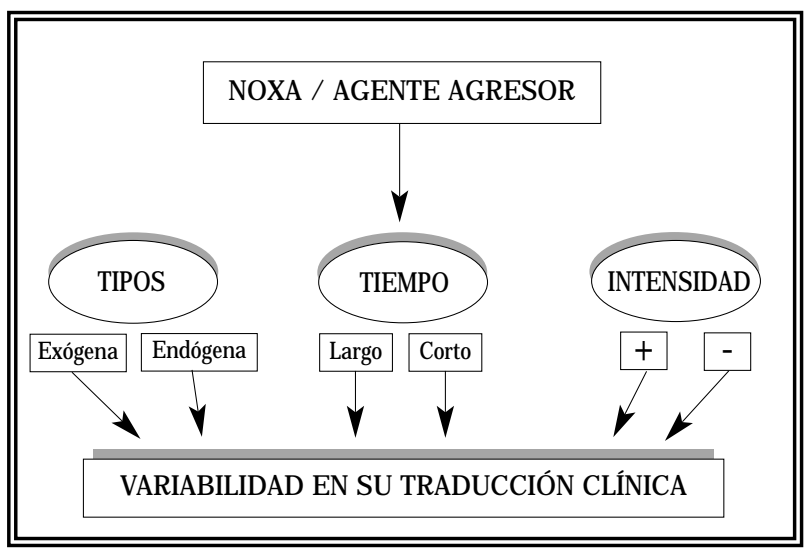

ciones metabólicas celulares, cambios en la expresión génica con su correspondiente traducción en la síntesis proteica y efectos moduladores directos sobre diversas poblaciones celulares, como por ejemplo ocurre con las endotoxinas de las bacterias gram negativas en las sepsis de origen urinario, sobre los polimorfos nucleares, las células accesorias e incluso sobre poblaciones linfocitarias.

En la modificación de la fisiología celular destacan la generación de radicales libres, la síntesis de proteínas de stress térmico y la expresión de genes de distintas citocinas o quimiocinas. La participación de estas moléculas posee un papel muy 
relevante en el fenómeno inflamatorio, ya que no sólo poseen capacidad de modulación de la activación, diferenciación y distribución tisular leucocitaria, sino que además, ejercen también actividades biológicas de regulación sobre células estructurales de diferentes órganos y sistemas incluidos los constitutivos de las estructuras genitourinarias.

Otra expresión de la modificación de la fisiología celular es la modificación del microambiente tisular como consecuencia de la presencia extracelular de estas moléculas.

Finalmente, la consecuencia de todos estos fenómenos es la aparición de un gradiente de señalización con repercusión más o menos inmediata en las células endoteliales de los vasos sanguíneos y de esta manera se comienza a favorecer la activación de células accesorias o fagocitos del sistema inmune, de linfocitos y de granulocitos.

La activación celular es ya un proceso activo de respuesta en el que la eficiencia es fundamental, ya que si no ocurre se comenzará a producir daño tisular. La actividad de respuesta fagocítica en este momento del proceso es determinante, ya que puede condicionar la acumulación material del agente agresor. En otras circunstancias, por el contrario, existirá una resistencia a la actividad enzimática de los gránulos de las células fagocíticas.

El proceso ulterior está representado por la presentación de los diferentes elementos constitutivos del agente agresor a la población de células $\mathrm{T}$ cooperadoras del sistema inmune, desencadenando una respuesta inmunológica secuencial.

La activación de los linfocitos T se complementa con amplificaciones de la respuesta frente a los antígenos responsables y activación de los linfocitos $\mathrm{T}$ citotóxicos y otros con diversas capacidades de reconocimiento. También, según las características antigénicas del agente agresor (fundamentalmente virus y otros microorganismos) se estimulan las células NK e incluso, las propias células fagocíticas, mononucleares y polinucleares tienen capacidad efectora de inducir la lisis de las células del organismo con componentes del agente etiológico o, incluso las propias, modificadas o no, en el entorno inflamatorio.

Todo el proceso celular mencionado es un proceso activo en el que se producen diversos pro- ductos metabólicos y citocinas con capacidad para modificar el ambiente tisular local. A este nivel es preciso destacar en la fase inicial de la respuesta inflamatoria, la liberación de quimioquinas, grupo de citocinas con capacidad moduladora de la distribución linfocitaria. Estas moléculas ejercen una función quimioactiva sobre los leucocitos, que se movilizan de acuerdo a su gradiente de concentración tisular. Las moléculas de adhesión y los propios receptores de las mencionadas quemocinas en las células endoteliales del ambiente tisular modifican su expresión e incrementan la adhesividad de la pared para los leucocitos circulantes. Éstos, además, están expuestos a la acción moduladora de las citocinas liberadas en el entorno inflamatorio y a la de algunos de los productos de los agentes etiológicos. El resultado final es el inicio de la adhesión leucocitaria a la pared del vaso y su emigración secuencial hacia el interior tisular, hacia el foco inflamatorio. De forma simultánea, los productos inflamatorios, tanto los de origen celular del individuo, como los correspondientes al agente agresor, penetran en los vasos linfáticos alcanzando a los tejidos linfoides locorregionales, iniciando su repercusión en el sistema linfoide de la mucosa o en los ganglios linfáticos próximos.

Otros componentes relevantes de la respuesta inflamatoria, son las enzimas proteolíticas correspondientes a los componentes citoplasmáticos celulares y los propios linfocitos B.

Los momentos evolutivos de la respuesta inflamatoria son secuenciales y los mecanismos de control condicionan, finalmente, su mayor o menor intensidad y el resultado final será la destrucción y anulación del agente etiológico o, de no ser eficiente en una unidad de tiempo determinado, contribuir a la amplificación del daño tisular.

La amplificación sistémica de la reacción inflamatoria también merece ser destacada, ya que traduce la acción general de las moléculas secretadas en el tejido o por las de la respuesta inflamatoria. En este sentido, es destacable que distintas citocinas como la interleucina 1 , la 6 y el factor de necrosis tumoral, tienen actividad moduladora sobre el sistema nervioso central modificando el funcionalismo neuronal y la secreción de neurotransmisores. También, es conocida la extremada relevancia de estas moléculas sobre los centros 
reguladores hipotalámicos y la generación de fiebre. Además, distintas células del organismo como los hepatocitos, el mensangio, células del parénquima pulmonar, etc. son susceptibles a la acción de diferentes citocinas y productos generados durante la inflamación. En concreto, la acción de la interleucina 6 y también de la propia interleucina $1 \mathrm{y}$ del factor de necrosis tumoral sobre los hepatocitos, modulan la síntesis de los denominados reactivos de fase aguda.

Razonablemente, la respuesta inflamatoria debe poseer un carácter temporal, en otra circunstancia su cronificación o perpetuación expresaría un modelo de respuesta ineficiente. Desde un punto de vista genérico, los modelos clínicos de respuesta inflamatoria pueden agruparse en eficacia clínica (anulación y destrucción del agente etiológico), ineficacia (generalización y expansión de la acción patógena) y múltiples situaciones clínicas que académicamente podemos considerar intermedias en las que no se produce una verdadera destrucción del mecanismo agresor y en las que lo que ocurre es la génesis de una modificación en el comportamiento biológico de las células inflamatorias adquiriendo un patrón de expresión génica y de producción de proteínas que modulan de forma directa la actividad de los fibroblastos y de las células endoteliales.

La traducción clínica de la última de las respuestas inflamatorias mencionadas es muy relevante, ya que en ella se asume una progresión hacia la cronificación definida por la proliferación vascular, la hiperplasia fibroblástica y la fibrosis. Esta respuesta inflamatoria supone un "equilibrio" de respuesta denominada "fase curativa" del colágeno y los vasos, y como se desarrollará ulteriormente, es aplicable a algunas enfermedades fibrosantes descritas en el aparato genitourinario.

\section{FENÓMENO INFLAMATORIO Y RESPUESTA CLÍNICA GE NERAL}

Las bases biológicas del fenómeno inflamatorio explican su carácter dinámico y la traducción clínica de estos complejos mecanismos secuenciales explican su pluralidad y la identificación de momentos evolutivos muy diferentes entre sí. Simultáneamente, otros acontecimientos biológicos son comunes y no se relacionan estrechamente con el agente agresor.
Un primer factor determinante a considerar para entender la respuesta inflamatoria es su localización topográfica. Los determinantes anatómicos poseen un papel trascendental, ya que el mecanismo lesional representado por el infiltrado celular, el aumento del espacio extracelular, el incremento de la permeabilidad de los vasos e incluso, en los estadios de fibrosis y de hiperplasia fibroblástica, la retracción del tejido, es diferente según que ocurra en órganos con mayor o menor perfusión (riñón...), distensibilidad (vejiga...) o accesibilidad por citar algunos ejemplos. En este sentido, cabe destacar la particular disposición del aparato genitourinario constituido por entramados sistemas canaliculares dinámicos e interrelacionados, de tal forma que la traducción clínica final es extremadamente órgano-dependiente, en base a las múltiples funciones de almacenamiento, conducción y transporte del aparato urinario.

Para poder explicar los modelos clínicos más representativos, es inevitable considerar la fase aguda del daño tisular con mecanismos efectores de daño celular y parenquimatoso propios y la fase de daño tisular con más o menos cronificación con mecanismos de regulación y activación de la respuesta inflamatoria de distinta naturaleza.

La traducción clínica en la fase aguda es rica en infiltrado celular, en producción de mediadores y en modificaciones del compartimento vascular; se trata de la fase más exudativa de la respuesta inflamatoria. Es propio de la respuesta a agentes de exposición temporal limitada; la naturaleza del agente agresor, en estas circunstancias, no es determinante (ej. un microorganismo o una litiasis). La acción sobre el epitelio de revestimiento del aparato urinario, en esta circunstancia, se traducirá en una respuesta definida por una infiltración celular rica en células polimorfonucleares y en células no nucleares, y por tanto, unas manifestaciones clínicas preferentemente irritativas asociadas al aumento de la permeabilidad celular y a la exudación celular.

En las circunstancias de perpetuación de la acción del agente agresor con respuesta inflamatoria ineficiente es posible también identificar una fase inicial de respuesta exudativa inflamatoria intensa, pero se tiende al equilibrio entre la persistencia de la respuesta inflamatoria y la progre- 
siva aparición de la denominada regeneración tisular fibroblástica. En este caso, el patrón de traducción clínica está dominado por síntomas que ocultan su verdadera naturaleza, la expresión sistémica puede ser poco relevante pero los fenómenos locales traducen la sustitución del tejido normal por los cambios fibróticos.

En definitiva, se puede deducir de todas estas afirmaciones que la clínica de la respuesta inflamatoria no es monomorfa, sino que es polimorfa, con componentes diferenciales característicos relacionados con la configuración anatómica y los estadios evolutivos tan diversos de esta respuesta. Finalmente, al considerar su repercusión en la elección terapéutica, deben contemplarse los objetivos de retirada / eliminación del agente etiológico o su inhibición si no fuera posible su erradicación. En este contexto, en fases precoces se debe procurar la inhibición de producción de los reactantes inflamatorios, su anulación funcional o, incluso, impedir la emigración celular. En las fases crónicas, por el contrario, se pretende anular el estado de equilibrio que favorece la proliferación fibroblástica y la fibrogénesis intentando romper el círculo vicioso de la respuesta inflamatoria mantenida.

Todas las circunstancias mencionadas permiten plantear una sistematización conceptual de la patología inflamatoria de localización genitourinaria, atendiendo preferentemente a la identificación de los agentes promotores del daño tisular y la consiguiente respuesta inflamatoria.

\section{AGENTES BIOLÓGICOS Y RESPUESTA TISULAR INFLAMATORIA GENITOURINARIA}

La trascendencia de la agresión mediada por agentes de naturaleza biológica en la patogenia de la respuesta inflamatoria, es muy relevante. Por un lado, sus mecanismos patogénicos cada vez más conocidos poseen rasgos, en muchos casos, comunes con independencia del agente agresor y su comprensión integra la consideración de los mecanismos defensivos del huésped; por otro lado, y de mayor relevancia en los objetivos de esta revisión, su traducción clínica posee clarísimas implicaciones asistenciales que abarcan modelos tanto de atención primaria (síndrome uretral, cistitis aguda...) como altamente especializadas (shock séptico).
Las situaciones clínicas que traducen la respuesta inflamatoria frente a agresiones por agentes biológicos corresponden a entidades anatomoclínicas concretas y muy variadas que se reflejan en la Tabla III.

\section{TABLA III}

AGRESIÓN BIOLÓGICA Y RESPUESTA INFLAMATORIA TISULAR. MODALIDADES CLÍNICAS*

- Síndrome uretral

- Bacteriuria asintomática

- Cistitis aguda bacteriana

- Nefropatías tubulointersticiales infecciosas.

- Prostatitis aguda (tipo I)

- Prostatitis crónica bacteriana (tipo II)

- Cistitis de repetición

- Infecciones específicas (mycobacterias, hongos...)

- Abscesos renales

- Pionefrosis

- Infecciones genitales

- Shock séptico (reacción inflamatoria sistémica)

*Con independencia del agente uropatógeno responsable y del carácter agudo o crónico

Es destacable el potencial de diseminación sistémica de la patología infecciosa a partir del aparato urinario con las características de una sepsis y su trascendencia en términos de morbimortalidad; asimismo, no debe ignorarse que las formas recidivantes de infección urinaria son las responsables de mantener todavía, en la actualidad, tasas de frecuencia muy elevadas, circunstancia que ocurre a pesar de disponer y haber incorporado múltiples y eficaces antibióticos en el arsenal terapéutico con estas indicaciones específicas y con una eficacia clínica universalmente aceptada

\section{AGENTES FÍSICOS, QUíMICOS Y MECÁNICOS Y RESPUESTA TISULAR INFLAMATORIA GE NITOURINARIA}

La agresión de órganos genitourinarios a través de mecanismos lesionales inductores de lesión inflamatoria incluye a agentes físicos, químicos y mecánicos propiamente dichos. Las circunstancias de daó tisular inducido por estos agentes poseen el denominador común de no precisar de 
otros mecanismos complementarios; poseen, por sí mismos, capacidad inductora de lesión de naturaleza inflamatoria.

Las peculiares características anatómicas y funcionales de la cavidad vesical explican que en la mayoría de las circunstancias clínicas en las que actúan estos agentes la infiltración inflamatoria y el daño tisular ocurra, predominantemente, en la vejiga. La doble circunstancia de órgano de almacenamiento y contacto de la orina con la mayor superficie urotelial subyacen como los elementos diana de toda la patogenia. En este contexto se pierde la capacidad funcional vesical, interfiriendo con su misión de aislamiento e impermeabilización de su estructura básica, a saber, la constituida por el estrato muscular.

La sistematización clínica de estos acontecimientos, obliga a reclamar el extenso abanico de las cistopatías, y en este caso concreto las inducidas por los mencionados agentes. Sin embargo, la inducción de la lesión inflamatoria no siempre posee como punto de partida inicial el contacto directo urotelial, sino que en otras circunstancias, como por ejemplo las inducidas por agentes físicos (irradiación), la exposición es mucho más extensa y abarca todos los elementos anatómicos constitutivos del aparato genitourinario, es decir, mucosa y submucosa urotelial (recubrimiento interno), estrato muscular y soporte conectivo adiposo que incluye los pedículos vasulonerviosos $\mathrm{y}$, además, en cualquier órgano o localización.

Globalmente considerada, la patología inflamatoria que traduce agresión por agentes de naturaleza física, química o mecánica, contemplaría la acción de sustancias y complejos moleculares que actuarian con finalidad terapéutica (hormonas, fármacos), y de agentes físicos que integran las radiaciones ionizantes, los agentes térmicos (termoterapia e hipertermia) y los efectos mecánicos inducidos por el razonamiento y la obstrucción cuando existe litiasis urinaria o presencia de cuerpos extraños en cualquier localización anatómica del aparato urinario.

La correlación clínica tras considerar los mecanismos lesionales y la diversidad de agentes implicados, se refleja en las entidades anatomo-clínicas contempladas en la Tabla IV.

Existen situaciones clínicas con daño tisular genitourinario y participación inflamatoria en

\section{TABLA IV}

\section{AGRESIÓN FÍSICA / QUÍMICA / MECÁNICA Y RESPUESTA INFLAMATORIA TISULAR MODALIDADES CLINICAS*}

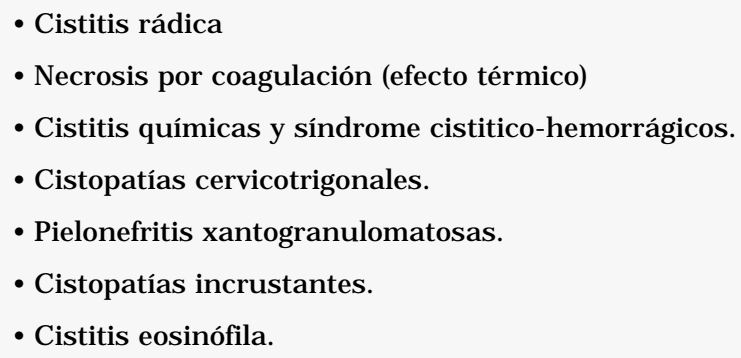

*Con independencia del agente y el carácter agudo o crónico.

mayor o menor intensidad, cuya etiología durante muchos años ha sido enigmática a pesar de tratarse de patologías frecuentes y tradicionales. El denominador común en muchas de ellas era precisamente su condición de patologías reto.

Actualmente, se puede asumir el considerable avance alcanzado, no sólo en la comprensión de diversos mecanismos de naturaleza inmunológica (autoinmunidad, etc...) identificados y asociados a diversas entidades clínicas como son las enfermedades del colágeno, las vasculitis, etc..., sino también, en el mayor conocimiento de los mecanismos de regulación del crecimiento y proliferación tisulares (apoptosis, necrosis celular, etc.)

La extrapolación e incorporación de algunos de estos avances biológicos a determinadas patologías genitourinarias de patogenia no aclarada, es una realidad, al menos parcialmente, y además posee el atractivo de contribuir a la comprensión de enfermedades cuyo enfoque terapéutico tradicionalmente resultaba desalentador.

La consideración de mecanismos patogénicos de carácter inmunológico, así como de las anomalías de la regulación del crecimiento y proliferación tisular, al menos parcialmente responsables de estas entidades clínicas, adquieren progresiva relevancia y es aplicable a la comprensión de diversos acontecimientos y situaciones clínicas que con la participación genitourinaria puede contemplarse por la propia implicación patológica con participación inmunológica, alteración del crecimiento celular, o por el contrario, por afectación secundaria en el contexto de enfermedades sistémicas autoinmunes. 
Las entidades clínico-urológicas susceptibles de integrarse en esta aproximación patogénica y su correspondiente respuesta inflamatoria se reflejan en la Tabla V.

\section{TABLA V}

DAÑO TISULAR CON PARTICIPACIÓN INFLAMATORIA, EN ÓRGANOS GENITOURINARIOS ASOCIADOS A MECANISMOS INMUNOLÓGICOS Y ANOMALÍAS DEL CRECIMIENTO CELULAR

\section{DAÑO TISULAR GENITOURINARIO INFLAMATORIO CON IMPLICACIÓN PATOGÉNICA AUTOINMUNE Y ALTERACIÓN DEL CRECIMIENTO CELULAR}

- ENFERMEDADES FIBROSANTES

- Fibrosis retroperitoneal idiopática

- Enfermedad de Peyronie

- CISTOPATÍAS

- Cistitis intersticial

- Cistitis folicular

- ENFERMEDADES PROSTÁTICAS

- Prostatitis y síndromes relacionados

- Hiperplasia benigna prostática

\section{- AFECTACIÓN GENITOURINARIA EN} ENFERMEDADES SISTÉMICAS AUTOINMUNES.

- Síndrome de Reiter

- Síndrome de Behçet

- Otros

- Lupus eritematoso

- Poliarteritis nudosa

- Granulomatosis de Wegener

- Púrpura de Schölein-Henoch

- Síndrome de Sjögren

\section{REFERENCIAS}

1. J CARBALLIDO RODRÍGUEZ, M ÁlVAREZ-MON SOTO, C OLIVIER GÓMEZ: Patología inflamatoria en urología. Aspectos patogénicos y terapéuticos. Tema Monográfico LXV Congreso Nacional de Urología. Ene Ediciones. Mayo 2000.

2. KENNETH E, MOORE KEITH J: Lookingland Proinflammatory Cytokines in WE Paul. Fundamental Immunology Fourth Edition pp Lippicott-Raven Publishers Philadelphia 1999.

3. WARD PA, LENTSCH AB: The acute inflammatory response and its regulation. Arch Surg 1999; Jun; 134 (6): 666-669.

4. GABAI C, KUSHNER I: Mechanism of disease: Acutephase proteins and other systemic responses to inflammation. $N$ Engl J Med 1999; 340: 448-454.

5. HF ROSENBERG, JJ GALLIN: Inflammation In WE Paul. Fundamental Immunology Fourth Edition pp Lippicott-Raven Publishers. Philadelphia 1999.

6. PRIETO A, REYES E, PÉREZ A, ÁLVAREZ MON M: Moléculas co-estimuladoras tanto solubles como de membrana implicadas en la presentación antigénica y la respuesta inmune. Medicine 1997; 7: 2.255-2.262.

7. SOBEL J: Pathogenesis of urinary tract infection. Infec Dis Clin North Am 1997; 531-544.

8. CIFUENTES DELATTE L: Cistitis y cistopatías (2Ed.) Bok S.A. (Ed). 1989; Madrid.

9. VICENTE J, ALGABA F: Semiología diagnóstica endovesical. Pulso Ediciones, S.A. Barcelona, 1998.

10. CARBALLIDO J, ÁLVAREZ-MON M, LEIVA O, DE LA FUENTE A, TEJIDO A, ZULOAGA A: Fibrosis retroperitoneal idiopática. Mecanismos patogénicos. En Urología y retroperitoneo. Zuloaga Gómez A, Ene Ediciones 1997; 6: 101-125. Madrid.

Dr. J. Carballido Rodríguez

Servicio de Urología

Clínica Puerta Hierro

San Martín de Porres, 4

28035 Madrid

(Trabajo recibido el 2 diciembre 2002) 